\title{
TRAIL-overexpressing Adipose Tissue-derived Mesenchymal Stem Cells Efficiently Inhibit Tumor Growth in an H460 Xenograft Model
}

\author{
YOUNG UN CHOI ${ }^{*}$, YONGDAE YOON ${ }^{2 *}$, PIL YOUNG JUNG ${ }^{1}$, SOONJAE HWANG ${ }^{2}$, JU EUN HONG $^{3}$, \\ WOO-SEUNG KIM ${ }^{3}$, JOON HYUNG SOHN ${ }^{4}$, KI-JONG RHEE ${ }^{3}$, KEUM SEOK BAE ${ }^{1}$ and YOUNG WOO EOM ${ }^{2,5}$ \\ ${ }^{1}$ Department of General Surgery, Yonsei University Wonju College of Medicine, Wonju, Republic of Korea; \\ ${ }^{2}$ Regeneration Medicine Research Center, Yonsei University Wonju College of Medicine, Wonju, Republic of Korea; \\ ${ }^{3}$ Department of Biomedical Laboratory Science, \\ Yonsei University Mirae Campus College of Health Sciences, Wonju, Republic of Korea; \\ ${ }^{4}$ Central Research Laboratory, Yonsei University Wonju College of Medicine, Wonju, Republic of Korea; \\ ${ }^{5}$ Cell Therapy and Tissue Engineering Center, Yonsei University Wonju College of Medicine, Wonju, Republic of Korea
}

\begin{abstract}
Background/Aim: Mesenchymal stem cell-based tumor therapy is still limited due to the insufficient secretion of effectors and discrepancies between their in vitro and in vivo efficacy. We investigated whether genetically engineered adipose tissue-derived mesenchymal stem cells (ASCs) overexpressing tumor necrosis factor-related apoptosis-inducing ligand (TRAIL) had inhibitory effects on H460 tumor growth both in vitro and in an 4460 xenograft model. Materials and Methods: Genetically engineered adipose tissue-derived mesenchymal stem cells (ASCs) overexpressing tumor necrosis factor-related apoptosis-inducing ligand (TRAIL) were obtained from plasmid transfection with $p C M V 3-T R A I L$ and -interferon (IFN)- $\beta$ (producing ASC-TRAIL and ASC-IFN- $\beta$, respectively). Death of H460 cells co-cultured with ASCs, ASC-TRAIL, and ASC-IFN$\beta$ or exposed to their conditioned medium was evaluated via apoptosis and cytotoxicity assays. In addition, in an $\mathrm{H} 460$ xenograft model ( $n=10$ per group), the antitumor potential of
\end{abstract}

This article is freely accessible online.

*These Authors contributed equally to this work.

Correspondence to: Keum Seok Bae, MD, Ph.D., Department of General Surgery, Yonsei University Wonju College of Medicine, 20 Ilsan-ro, Wonju, Gangwon-do 26426, Republic of Korea. Tel: +82 337410573, e-mail: bksgs@yonsei.ac.kr and Young Woo Eom, Ph.D., Cell Therapy and Tissue Engineering Center, Yonsei University Wonju College of Medicine, 20 Ilsan-ro, Wonju, Gangwon-do, 26426, Republic of Korea. Tel: +82 337410260, e-mail: yweom@yonsei.ac.kr

Key Words: Mesenchymal stem cells, tumor necrosis factor-related apoptosis-inducing ligand, interferon- $\beta$, genetic engineering.
TRAIL-overexpressing, and IFN- $\beta$-overexpressing ASCs was investigated. Results: Conditioned medium obtained from ASCIFN- $\beta$ increased apoptosis of $H 460$ cells more than did ASCTRAIL. Additionally, in H460 xenograft models, while native ASCs promoted tumor growth, ASC-TRAIL and ASC-IFN- $\beta$ both dramatically suppressed tumor growth. Interestingly, in the context of ASC-IFN- $\beta$, tumors were detected only in $20 \%$ of nude mice, with smaller sizes and lower weights than those of the control group. Conclusion: TRAIL-overexpressing ASCs can be used to treat tumors; ASC-IFN- $\beta$ in particular secrete a higher level of TRAIL.

Mesenchymal stem cells (MSCs) have the potential to migrate and repair damaged tissues and wounds, including tumors, which are considered as wounds that do not heal (13 ). However, in the tumor microenvironment, MSCs can act as a double-edged sword, either promoting or suppressing tumor growth $(4,5)$. Numerous mediators secreted by MSCs are pro-tumorigenic: Immunosuppressive agents, angiogenic agents, mediators of the transition of MSCs into cancerassociated fibroblasts, epithelial-mesenchymal transitionpromoting agents, and tumor metastasis-promoting agents (5, $6)$. In contrast, MSCs have also been reported to prevent tumor growth through anti-angiogenesis, cell-cycle arrest and apoptosis, down-regulation of survival signaling, and immune cell infiltration $(7,8)$.

Despite the anti-tumorigenic potential of MSCs, MSC-based tumor therapy is still limited due to the insufficient secretion of effector molecules and discrepancies between their in vitro and in vivo efficacy (8). To overcome these limitations, genetic modifications of MSCs have been proposed (9-11). Tumor necrosis factor-related apoptosis-inducing ligand (TRAIL) can specifically induce apoptosis of tumor cells via binding to its 
cognate transmembrane death receptors, TRAIL-R1 or TRAIL-R2. In fact, TRAIL has already been overexpressed in MSCs for tumor treatment approaches $(12,13)$. Additionally, the overexpression of interferons (IFNs) in MSCs can also lead to the up-regulation of TRAIL $(14,15)$. IFNs comprise two main families, type I (IFN- $\alpha,-\beta,-\varepsilon,-\mathrm{K}$, and $-\omega$ ) and type II (IFN- $\gamma)(16)$, and induce the expression of IFN-stimulated genes, thereby leading to alterations in the state of differentiation, rate of proliferation, and functional activity of endothelial cells, immune effector cells and tumor cells. Of note, in melanoma and other cell lines, IFNs have been shown to activate the extrinsic apoptotic cascade through TRAIL expression $(15,17,18)$.

In previous work, we reported that adipose tissue-derived mesenchymal stem cells (ASCs) cultured at high density expressed IFN- $\beta$ and TRAIL and suppressed the growth of H460 human lung cancer cells (8). However, although ASCs expressing IFN- $\beta$ and TRAIL induced dramatic cell death of H460 cells in vitro, their tumor regression activity was not sufficient in vivo in the xenograft model (8). Here, we investigated whether genetically engineered MSCs overexpressing TRAIL (transfected with the pCMV3TRAIL/pCMV3-IFN- $\beta$ ) exert a suppressive effect on tumor growth and which line (TRAIL- or IFN- $\beta$-overexpressing cells) shows greater antitumor potential in vitro and in vivo.

\section{Materials and Methods}

Cell culture. This study was approved by the Institutional Review Board of Yonsei University Wonju College of Medicine (CR319048). Human adipose tissues from three healthy donors (24-38 years of age) who provided written-informed consent were obtained through elective liposuction procedures under anesthesia at the Wonju Severance Christian Hospital (Wonju, Republic of Korea). ASCs were isolated using a modified protocol described by Zuk et al. (19). Briefly, after removing contaminated blood cells and local anesthetics via a washing step with phosphate-buffered saline (PBS), adipose tissues were digested with $0.075 \%$ type IA collagenase (Sigma-Aldrich, St. Louis, MO, USA) in PBS to obtain mononuclear cells. Mononuclear cells $\left(5 \times 10^{6}\right)$ were then seeded in $100-\mathrm{mm}$ culture dishes with low-glucose Dulbecco's modified Eagle's medium (DMEM; Gibco, Rockville, MD, USA) containing 10\% fetal bovine serum (FBS; Gibco) and penicillin/streptomycin. After 2 days, the non-adherent cells were removed by changing the media and adherent cells were considered to be ASCs. Cells from passages $3-5$ were used in all experiments. To confirm that IFN- $\beta$ was able to induce TRAIL expression in ASCs, ASCs were exposed to 1000 $\mathrm{U} / \mathrm{ml}$ of IFN- $\beta$ for 2 days with or without Janus kinase 1 (JAK1) inhibitor $(100 \mathrm{nM})$ or JAK2 inhibitor $(20 \mu \mathrm{g} / \mathrm{ml})$.

The human lung cancer cell line H460 was purchased from the Korean Cell Line Bank (Seoul, Republic of Korea). H460 cells were maintained in DMEM (Gibco) supplemented with 10\% FBS and penicillin/streptomycin. For indirect co-culture, H460 cells and ASCs were seeded on lower and upper chambers of Transwell plates (Corning, Corning, NY, USA), respectively, and co-cultured for 2 days.
Transfection of ASCs. X-tremeGENE HP DNA transfection reagent (Roche, Basel, Switzerland) was used for gene editing of ASCs. Briefly, ASCs were seeded in 100-mm culture dishes at a density of $5.2 \times 10^{5}$ cells and cultured for $24 \mathrm{~h}$. Ten micrograms of pCMV3TRAIL or pCMV3-IFN- $\beta$ plasmid DNA (SinoBiological, Wayne, PA, USA) were diluted with $1 \mathrm{ml}$ of serum-free DMEM and reacted with $20 \mu \mathrm{l}$ of the $\mathrm{X}$-tremeGENE HP reagent for $20 \mathrm{~min}$ at $20-25^{\circ} \mathrm{C}$. The transfection complex was then added to the cells in a dropwise manner. Cells were incubated for 3 days, and then the conditioned medium $(\mathrm{CM})$ was recovered by centrifugation and stored at $-80^{\circ} \mathrm{C}$ until further analysis. The transfection efficiency of the X-tremeGENE HP reagent was compared to that in the context of Lipofectamine 2000 (Invitrogen, Eugene, OR, USA) using the pCMV3-green fluorescence protein (GFP) plasmid (SinoBiological); GFP expression was evaluated by flow cytometry 3 days post-transfection.

3-(4,5-Dimethylthiazol-2-yl)-2,5-diphenyltetrazolium bromide (MTT) assay. The $\mathrm{H} 460$ cells were plated at a density of $1 \times 10^{4}$ cells $/ \mathrm{cm}^{2}$ in 96 -well plates. After $24 \mathrm{~h}$, the cells were treated with recombinant TRAIL (1-100 ng/ml; R\&D Systems, Minneapolis, MN, USA) and IFN- $\beta$ (10-1000 U/ml; R\&D Systems) or CM for an additional $48 \mathrm{~h}$ and then MTT (Sigma, St. Louis, MO, USA) dissolved in PBS was added to each well (final concentration, 5 $\mathrm{mg} / \mathrm{ml}$ ) and plates were incubated at $37^{\circ} \mathrm{C}$ for $2 \mathrm{~h}$. The MTT formazan crystals formed were then dissolved in $100 \mu$ l dimethyl sulfoxide under agitation for $15 \mathrm{~min}$ and the optical density was then determined at $570 \mathrm{~nm}$ using a microplate reader (Molecular Devices, San Jose, CA, USA).

Immunoblotting. Proteins were extracted separately from the cells and $\mathrm{CM}$. The cells were lysed in sodium dodecyl sulfate-polyacrylamide gel electrophoresis (SDS-PAGE) sample buffer (62.5 mM Tris-HCl, pH $6.8 ; 1 \%$ SDS; $10 \%$ glycerol; and $5 \% \beta$-mercaptoethanol), and the $\mathrm{CM}$ was mixed $(1: 1)$ with $2 \times$ sample buffer. Protein samples were boiled for $5 \mathrm{~min}$, subjected to SDS-PAGE, and transferred to Immobilon polyvinylidene difluoride membranes (Millipore, Billerica, MA, USA). The membranes were blocked with $5 \%$ skim milk in Tris- $\mathrm{HCl}$ buffered saline containing $0.05 \%$ Tween 20 and then incubated with primary antibodies against signal transducer and activator of transcription 1 (STAT1), phospho (p)-STAT1, caspase-3, cleaved caspase-3 (1:2,000; Cell Signaling Technology, Danvers, MA, USA), TRAIL (1:1,000; R\&D Systems, Minneapolis, MN, USA), IFN- $\beta$, and glyceraldehyde 3-phosphate dehydrogenase $(1: 1,000$; Santa Cruz Biotechnology, Dallas, TX, USA), followed by peroxidase-conjugated secondary antibodies (1:2,000; Santa Cruz Biotechnology). The membranes were treated with EZ-Western Lumi Pico or Femto (DOGEN, Seoul, Republic of Korea) and visualized using the ChemiDoc XRS+ system (Bio-Rad, Hercules, CA, USA).

Apoptosis assay. Phycoerythrin-Annexin-V Apoptosis Detection Kit I (BD Biosciences, San Jose, CA, USA) was used according to the manufacturer's instructions. Briefly, cells were trypsinized, washed twice with cold PBS, and re-suspended in binding buffer. Cells were stained with phycoerythrin-annexin- $\mathrm{V}$ and 7-amino-actinomycin D for $15 \mathrm{~min}$ at room temperature in the dark and then analyzed without washing using a flow cytometer (BD FACSAria III) within $1 \mathrm{~h}$.

Animal studies. All animal experiments were performed in accordance with the institutional guidelines and approved by the Institutional Animal Care and Use Committee of the Yonsei University Mirae 
Campus, Wonju (YWCI-201911-009). Five-week-old athymic nude mice $(1520 \mathrm{~g})$ were purchased from Central Lab Animal Inc. (Seoul, Republic of Korea). To completely remove FBS from cells, H460 or ASCs were washed with PBS three times, once with Hank's balanced salt solution (HBSS; Sigma-Aldrich), and then re-suspended in HBSS for transplantation into nude mice. H460 $\left(2 \times 10^{6}\right.$ cells/mice $)$ and ASCs $\left(2 \times 10^{6}\right.$ cells/mice $)$ were mixed immediately prior to co-injection. Cells were then suspended in $100 \mu \mathrm{l}$ of HBSS and subcutaneously injected into the flanks of nude mice ( $\mathrm{n}=10$ per group). Sham groups were injected only with $\mathrm{H} 460$ cells $\left(2 \times 10^{6}\right.$ cells/mouse). Mice were examined three times weekly, and the tumor volumes $(\mathrm{L} \times \mathrm{W} \times \mathrm{H} \times 0.52$ $\mathrm{mm}^{3}$ ) were measured weekly using Vernier calipers for 3 weeks. Mice were then sacrificed by cervical dislocation; thereafter, the tumors were excised and photographed, and the tumor weights were measured using an electronic scale.

Statistical analysis. Data are presented as the mean \pm standard deviation or standard error of the mean. To compare group means, Student's $t$-test and one-way analysis of variance followed by Scheffe's test were performed. Statistical significance was set at $p<0.05$.

\section{Results}

Efficiency of transfection of ASCs with plasmid vectors. Viral vectors are mostly used for the genetic engineering of MSCs; however, safety issues related to the integration of the genetic material of viral vectors within the human genome (and potential for mutagenicity) as well as regarding the potential immunogenicity of viral antigens are emerging $(20,21)$. Therefore, in this study, we transfected MSCs with a non-viral plasmid vector (pCMV3-GFP) using lipid-based transfection reagents (Lipofectamine 2000 and X-tremeGENE HP) and evaluated the transfection efficiency by flow cytometry. On the second day after transfection, $10.61 \pm 2.42 \%$ of cells were GFPpositive in the context of Lipofectamine 2000, while $46.71 \pm 3.19 \%$ cells were GFP-positive in the context of the XtremeGENE HP reagent (Figure 1A). Therefore, the $\mathrm{X}$ tremeGENE HP reagent was used to obtain genetically engineered ASCs. ASCs were then transfected with pCMV3TRAIL or pCMV3-IFN- $\beta$ plasmids (ASC-TRAIL or ASC-IFN$\beta$, respectively), and the expression of TRAIL and IFN- $\beta$ was detected by immunoblotting. TRAIL was expressed in ASCTRAIL, and both TRAIL and IFN- $\beta$ were detected in ASC-IFN$\beta$ (Figure 1B). Interestingly, the level of TRAIL secreted in ASCIFN- $\beta$ was higher than in ASC-TRAIL, although the cellular TRAIL expression was similar in both cell types (Figure 1B). Previously, we reported that TRAIL expression in ASCs is IFN$\beta$-dependent (8). Here, ASCs exposed to exogenous IFN- $\beta$ dramatically increased the TRAIL expression level (Figure 1C). Interestingly, JAK1 inhibitors down-regulated the expression of TRAIL via blockage of the phosphorylation of STAT1. In conclusion, overall, we show that approximately $50 \%$ of ASCs were transfected with non-viral plasmids (pCMV-GFP) using the $\mathrm{X}$-tremeGENE HP reagent, and the expression of TRAIL was approximately $32 \%$ higher in ASC-IFN- $\beta$ than in ASC-TRAIL.
In vitro antitumor effects of ASCs overexpressing TRAIL. Next, to confirm whether ASCs overexpressing TRAIL are cytotoxic to H460 lung cancer cells, H460 cells were treated with CM obtained from 2-day cultures of ASC-TRAIL and ASC-IFN- $\beta$. Remarkably, CM from ASC-TRAIL and ASCIFN- $\beta$ induced approximately $42.65 \% \pm 0.04 \%$ and $55.76 \pm 0.05 \%$ cytotoxicity in $\mathrm{H} 460$ cells, respectively (Figure 2A). Although $\mathrm{H} 460$ cells were sensitive to exogenous TRAIL in a dose-dependent manner, $1,000 \mathrm{U} / \mathrm{ml}$ of IFN- $\beta$ led to led to approximately $13.3 \%$ cytotoxicity in $\mathrm{H} 460$ cells (Figure 2B). Therefore, CM obtained from both ASC-TRAIL and ASC-IFN- $\beta$ induced cytotoxicity in the context of H460 cells in a TRAIL-dependent manner. Next, we examined the rate of cell death of $\mathrm{H} 460$ cells after indirect co-culture with ASCs, ASC-TRAIL, and ASC-IFN- $\beta$ for 2 days. ASCTRAIL and ASC-IFN- $\beta$ increased the frequency of annexinV-positive/7-amino-actinomycin D-positive H460 cells (dead cells). Interestingly, ASC-IFN- $\beta$ had a greater effect than ASC-TRAIL (Figure 3A). In addition, cleaved caspase-3 was highly expressed in $\mathrm{H} 460$ cells treated with CM from ASCIFN- $\beta$; again, the expression was higher than that using $\mathrm{CM}$ from ASCs or ASC-TRAIL (Figure 3B). Overall, our results suggest that ASCs overexpressing TRAIL induce apoptosis of H460 cells and that the antitumor activity of ASCs can be increased via transfection with IFN- $\beta$.

Antitumor effects of ASCs overexpressing TRAIL in a xenograft animal model. Previously, we reported that although ASCs cultured at high density were able to kill H460 lung cancer cells effectively in vitro in a TRAILdependent manner, the inhibition of tumor growth was not significant in a xenograft animal model (8). Here, to investigate whether the TRAIL expression level influences the antitumor activity of ASCs, nude mice ( $n=10$ per group) were injected with $\mathrm{H} 460$ cells alone or together with ASCs, ASC-TRAIL, or ASC-IFN- $\beta$ subcutaneously. In nude mice transplanted with $\mathrm{H} 460$, tumor volume continued to increase with time. The tumor volumes were higher in the group transplanted with ASCs and H460 cells, but importantly, the tumor volumes were significantly lower in the groups receiving $\mathrm{H} 460$ with ASC-TRAIL or ASC-IFN- $\beta$ than in H460 cells alone (Figure 4A). In particular, the tumor volume did not increase in the H460 + ASC-IFN- $\beta$ injection group and was the smallest on the $21 \mathrm{st}$ day $(35.53 \pm 29.01$ $\mathrm{mm} 3$ ). On the $21 \mathrm{st}$ day after transplantation, the mice were euthanized, and the tumor weight was measured. The average tumor weight in the $\mathrm{H} 460$ tumor-bearing mice treated with ASC was approximately $73 \%$ heavier than the H460 control group. On the other hand, the average tumor weight in mice receiving ASC-TRAIL was $73 \%$ of the sham control, and was even lower in that receiving ASC-IFN- $\beta$ $(\sim 7 \%)$ (Figure $4 \mathrm{~B})$. Of note, the injection of $\mathrm{H} 460$ cells with ASC-TRAIL generated tumors in 8 out of 10 experimental 

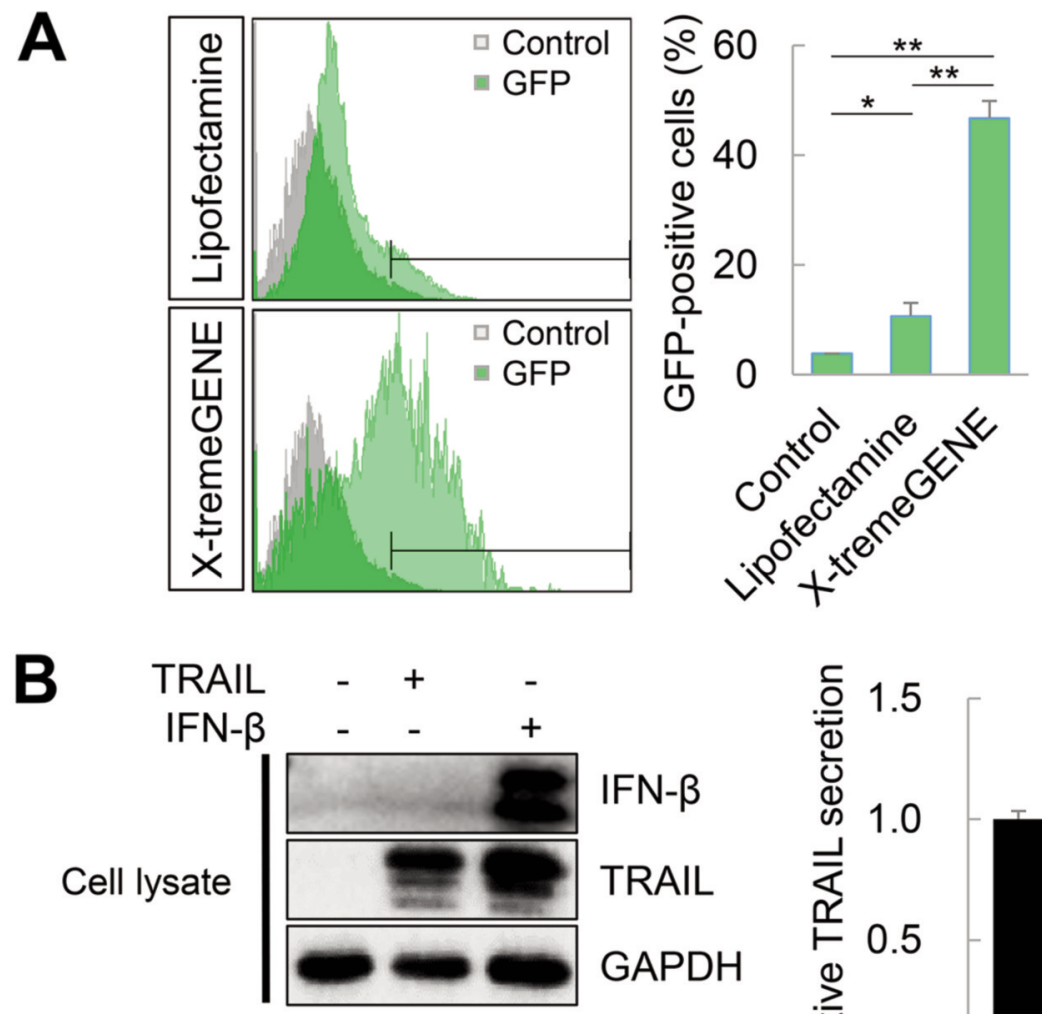

Culture sup.

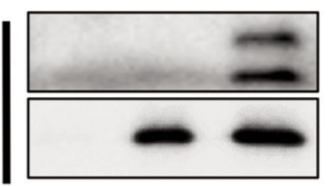

IFN- $\beta$

TRAIL
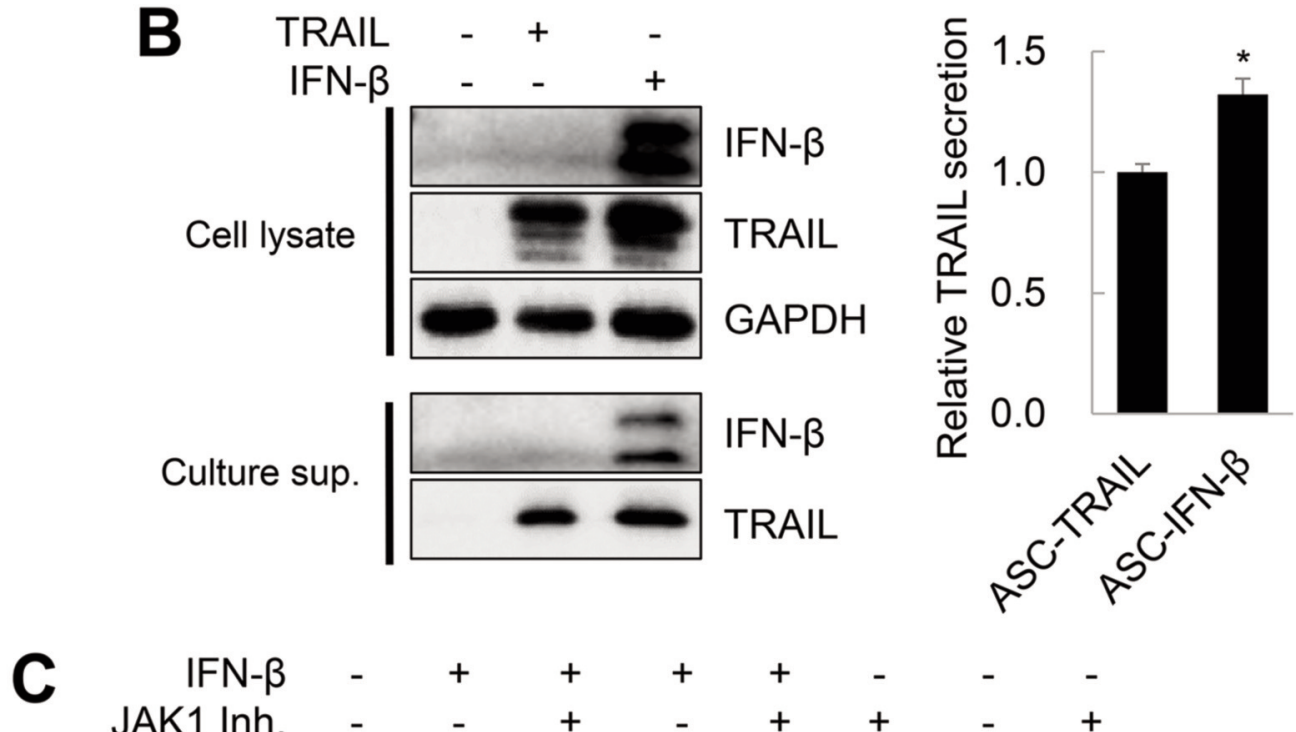

$\begin{array}{rrrrrrrrr}\text { IFN- } \beta & - & + & + & + & + & - & - & - \\ \text { JAK1 Inh. } & - & - & + & - & + & + & - & + \\ \text { JAK2 Inh. } & - & - & - & + & + & - & + & +\end{array}$

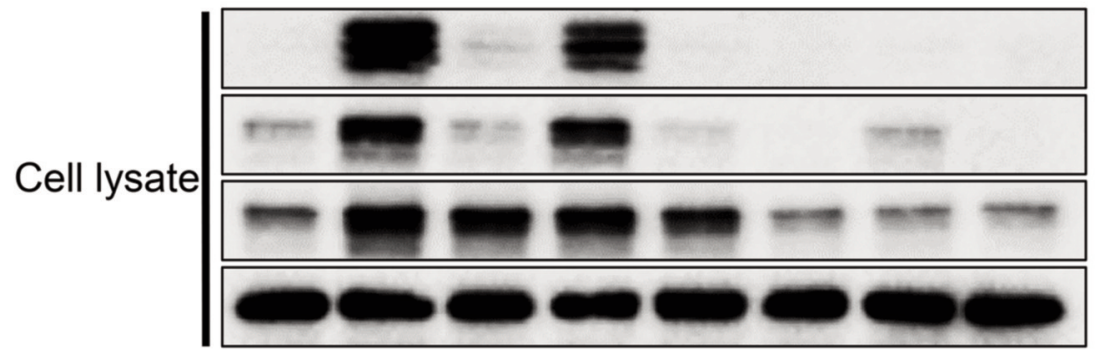

TRAIL

PSTAT1

STAT1

GAPDH

Culture sup.

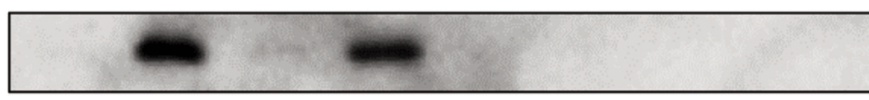

TRAIL

Figure 1. Transfection efficiency and tumor necrosis factor-related apoptosis-inducing ligand (TRAIL) overexpression in adipose tissue-derived mesenchymal stem cells (ASCs) transfected with plasmid vectors. A: Transfection efficiency in ASCs treated with the pCMV3-green fluorescence protein (GFP) plasmid and Lipofectamine 2000 or X-tremeGene HP. The frequency of GFP-positive cells was analyzed by flow cytometry. The data are expressed as the mean $\pm S D$ from three independent experiments. B: TRAIL-overexpressing ASCs. ASCs were transfected with $p C M V 3-T R A I L$ or $p C M V 3-$ interferon (IFN)- $\beta$ using X-tremeGENE HP reagent and the TRAIL expression levels were determined. Data were obtained from one of three independent experiments. C: TRAIL expression induced by exogenous IFN- $\beta$. ASCs were treated with exogenous IFN- $\beta$ with/without Janus kinase (JAK) $1 / 2$ inhibitors to understand whether the TRAIL expression was dependent on the JAK/signal transducer and activator of transcription (STAT) pathways. The TRAIL expression was then determined. Data were obtained from one of three independent experiments. Significantly different at: * $p \leq 0.05$ and $* * p \leq 0.01$. 
A

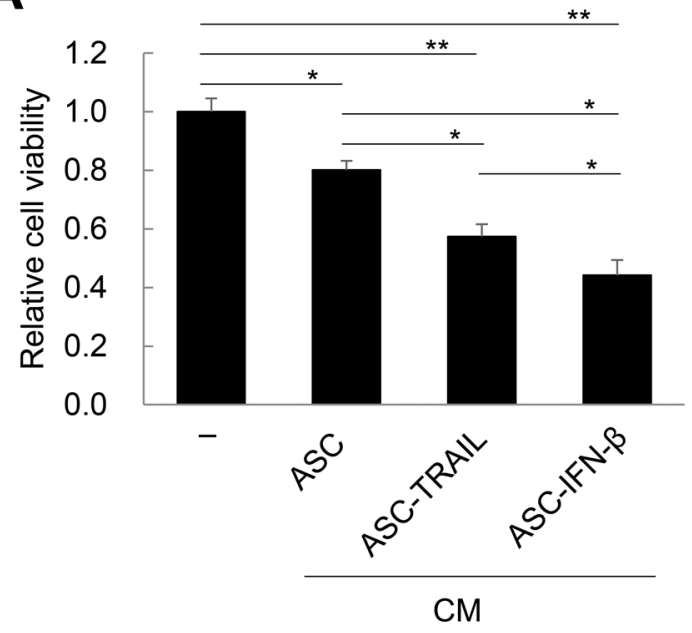

B

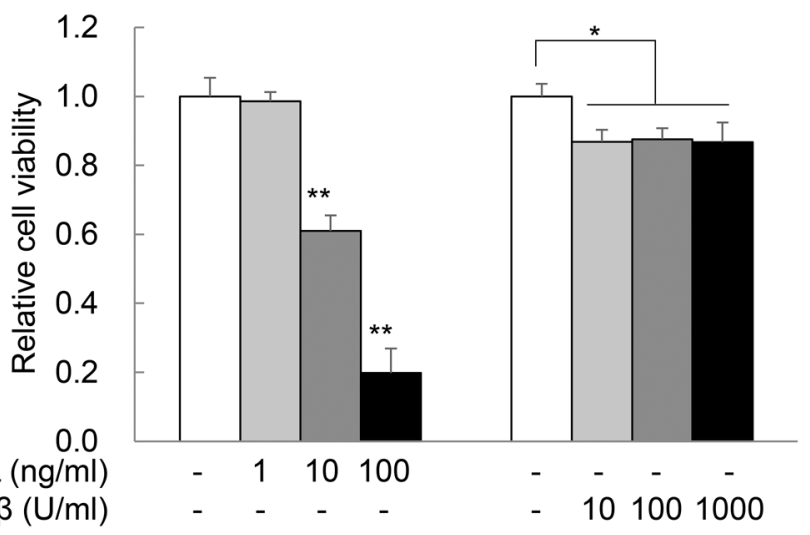

Figure 2. Cytotoxicity of H460 cells induced by tumor necrosis factor-related apoptosis-inducing ligand (TRAIL) and interferon (IFN)- $\beta$. A: Cytotoxicity of $H 460$ cells treated with conditioned media obtained from adipose tissue-derived mesenchymal stem cells (ASCs), ASC-TRAIL, or ASC-IFN- $\beta$ for 2 days. Cell viability was determined using the 3-(4,5-dimethylthiazol-2-yl)-2,5-diphenyltetrazolium bromide (MTT) assay. The data are expressed as the mean \pm SD from three independent experiments. B: Cytotoxicity of H460 cells treated with different doses of TRAIL and IFN- $\beta$ for 2 days. Cell viability was determined using the MTT assay. The data are expressed as the mean \pm SD from three independent experiments. Significantly different at: *p $\leq 0.05$ and $* * p \leq 0.01$.

mice, while that of $\mathrm{H} 460$ cells with ASC-IFN- $\beta$ generated tumors in only 2 out of 10 experimental mice (Figure 4C). These results suggest that TRAIL-overexpressing ASCs can inhibit tumorigenesis and tumor growth in xenograft models; in particular, IFN- $\beta$-transfected ASCs, with higher TRAIL expression, exhibit remarkable antitumor effects.

\section{Discussion}

Although the tumorigenic potential of MSCs has yet to be demonstrated in the context of clinical trials $(22,23)$, it has been reported that MSCs with tumor tropism can promote tumor progression or, conversely, suppress tumor growth $(4,5,7)$. In this study, we observed that ASCs overexpressing TRAIL induced apoptosis of $\mathrm{H} 460$ cells and efficiently controlled tumor growth, even in a xenograft model. In particular, ASCs transformed with IFN- $\beta$ secreted approximately $30 \%$ more TRAIL than ASCs transformed with TRAIL; importantly, in line with these results, ASC-IFN- $\beta$ suppressed tumor growth more efficiently than ASC-TRAIL both in vitro and in vivo.

Type I IFNs are pleiotropic cytokines that regulate many different cellular functions, including antiviral, antiproliferative, and immunoregulatory responses (24). In particular, IFN- $\beta$ is known to lead preferentially to the expression of TRAIL $(15,25)$. Previously, we reported that when ASCs were cultured at high density, type I IFNs and TRAIL were expressed $(8,26)$. In addition, IFN- $\beta$ mRNA was also expressed under serum-depleted conditions. Of note,
ASCs cultured at high density effectively induced cytotoxicity of H460 cells through TRAIL in vitro but without a tumorsuppressive effect in vivo (8). On the other hand, here we show that ASCs transformed with IFN- $\beta$ or TRAIL overexpression plasmids had a high tumor-suppressive effect in both in vitro and in vivo models; in particular, ASC-IFN- $\beta$ effectively controlled tumor growth in vivo. The difference between these two studies may be due to differences in the TRAIL expression levels. Overall, our results suggest that in order to use MSCs as antitumor agents, the overexpression of TRAIL through genetic modification is probably needed to maximize their tumor-suppressive potential.

Viral and non-viral vectors are used to deliver genes to MSCs. Since MSCs are intended for clinical application, safety must be the first priority during the selection of vectors. Gene delivery using viral vectors allows stable expression with high efficiency; however, potential immunogenicity and insertional mutagenesis are two potential limitations of such vectors $(20,21)$. In fact, it has been reported that hematopoietic stem cells engineered with a retroviral vector developed leukemia through insertional mutagenic transformation in four patients (27). In contrast, although non-viral vectors have a low transfection efficiency and induce transient gene expression, they are associated with relatively few side-effects. Recently, to improve the efficiency of non-viral vectors, single or combinations of cationic lipids, surfactants, peptides, polysaccharides, nanoparticles (gold, magnetic iron), and synthetic polymers 
A
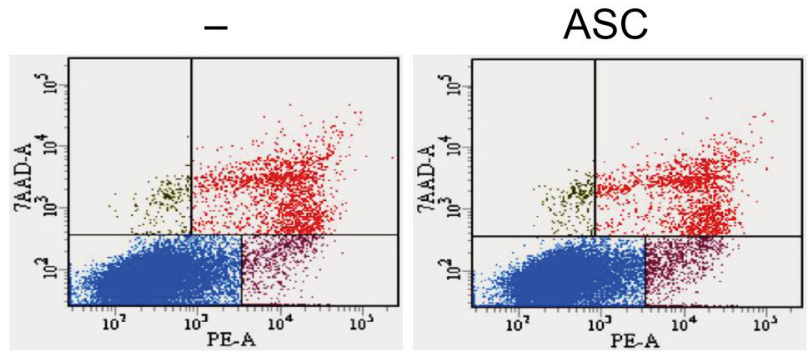

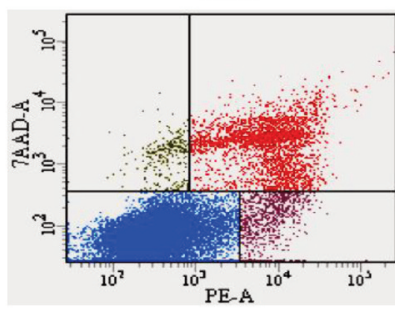

ASC-TRAIL

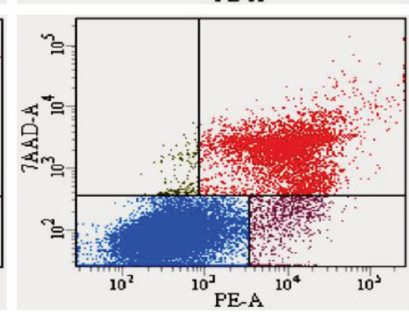

ASC-IFN- $\beta$

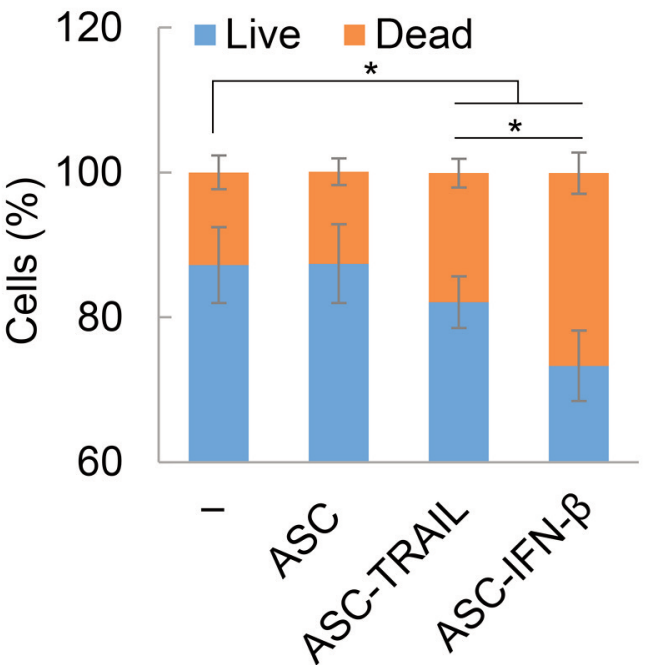

B
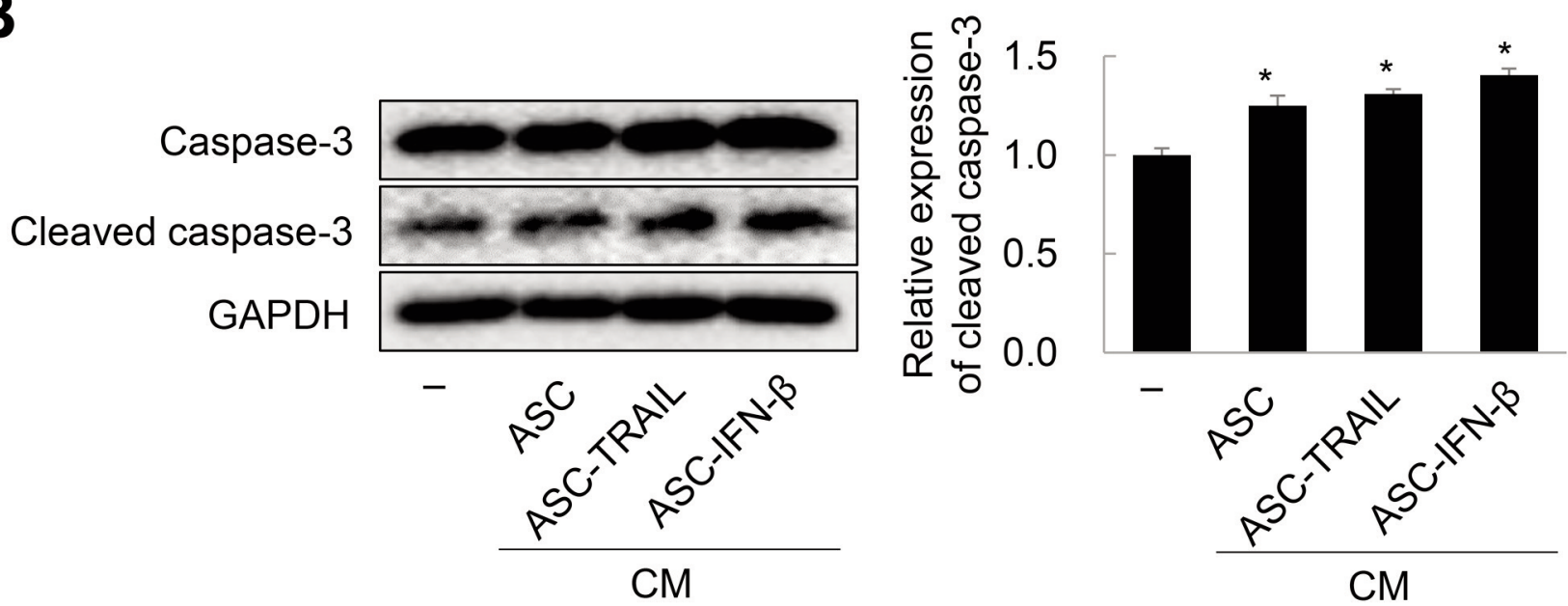

Figure 3. Death of $H 460$ cells exposed to adipose tissue-derived mesenchymal stem cells (ASCs) overexpressing tumor necrosis factor-related apoptosis-inducing ligand (TRAIL), ASC-interferon (IFN)- $\beta$, or their conditioned medium (CM). A: Death of H460 cells indirectly co-cultured with ASCs, ASC-TRAIL, or ASC-IFN- $\beta$ for 2 days was evaluated via phycoerythrin (PE)-annexin-V and 7-amino-actinomycin D (7-AAD) staining. Annexin-V-positive/7-AAD-negative, annexin-V-positive/7-AAD-positive, and annexin-V-negative/7-AAD-positive cells were considered dead cells. Data are expressed as the mean $\pm S D$ from three independent experiments. B: Caspase-3 activation in H460 cells treated with the CM from ASCs, ASC-TRAIL, and ASC-IFN- $\beta$ for 2 days. The expression of cleaved caspase-3 was detected by immunoblotting. The data are expressed as the mean $\pm S D$ from three independent experiments. Significantly different at: $* p \leq 0.05$.

have been used $(11,28,29)$. Therefore, it is essential to choose the optimal vector (depending on the disease condition to be treated) and to develop new techniques to compensate for the disadvantages of non-viral tools. If such studies are conducted in the future, it is expected that the therapeutic efficacy of MSCs will improve, as will consequently the outcomes of various diseases and, ultimately, the quality of life of patients.
In conclusion, our results suggest that genetically engineered TRAIL-overexpressing ASCs can be used to treat tumors, particularly, ASC expressing IFN- $\beta$, which secrete a higher level of TRAIL.

\section{Conflicts of Interest}

The Authors declare no conflicts of interest regarding this study. 

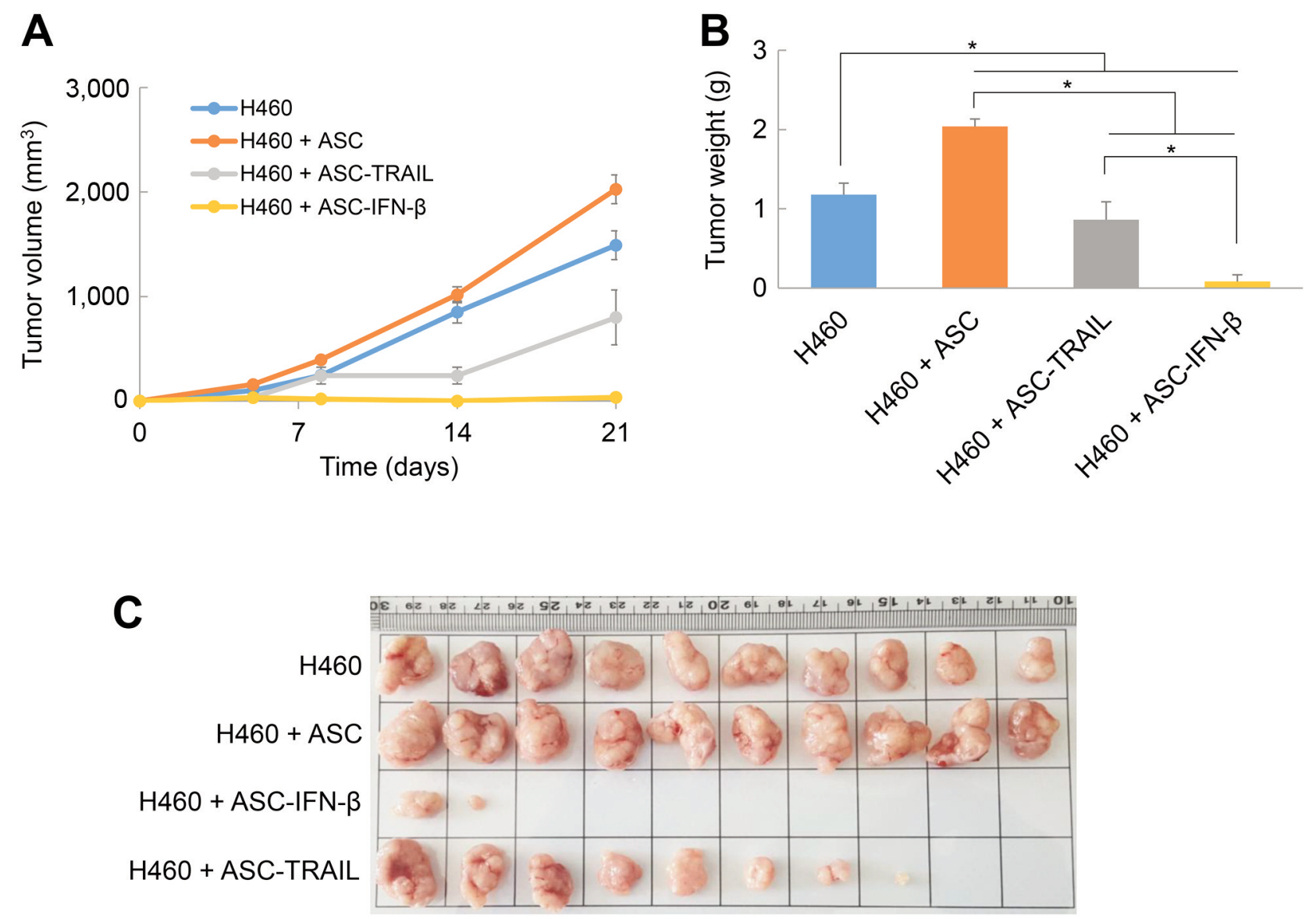

Figure 4. Antitumor effects of adipose tissue-derived mesenchymal stem cells (ASCs) overexpressing tumor necrosis factor-related apoptosis-inducing ligand (TRAIL) in xenograft animal models. A: Changes in the tumor volume of mice after ASC transplantation. The tumor volume was measured using Vernier calipers at the indicated time-points (for 3 weeks) after the transplantation of H460 cells alone, or together with ASCs, ASC-TRAIL, and ASC-interferon (IFN)- $\beta$. The data are expressed as the mean $\pm S E(n=10$ per group). B: Tumor weight 3 weeks after the transplantation of H460 cells alone, and together with ASCs, ASC-TRAIL, or ASC-IFN- $\beta$. The data are expressed as the mean $\pm S E$ ( $n=10$ per group). C: Tumor morphology. Three weeks after the injection of $H 460$ cells alone, or together with ASCs, ASC-TRAIL, and ASC-IFN- $\beta$, the mice ( $n=10$ per group) were sacrificed by cervical dislocation and the tumor masses were separated and photographed. Significantly different at $* p \leq 0.05$.

\section{Authors' Contributions}

Study concepts and study design, data analysis and interpretation, quality control of data and algorithms: Young Un Choi, Yongdae Yoon, Keum Seok Bae and Young Woo Eom. Data acquisition: Yongdae Yoon, Soonjae Hwang, Joon Hyung Sohn, Ju Eun Hong and WooSeung Kim. Investigation: Young Un Choi, Yongdae Yoon and Young Woo Eom. Statistical analysis, visualization of the results: Pil Young Jung, Soonjae Hwang and Ki-Jong Rhee. Article preparation: Young Woo Eom. Article editing and review: Young Un Choi, Keum Seok Bae and Young Woo Eom. All Authors approved the final article.

\section{Acknowledgements}

This work was supported by a grant from the Korea Health Technology R\&D Project through the Korea Health Industry Development Institute (KHIDI), funded by the Ministry of Health
\& Welfare, Republic of Korea (HI17C1365), and by the Basic Science Research Program through the National Research Foundation of Korea (NRF), funded the Ministry of Education, Republic of Korea (NRF-2017R1D1A1A02019212).

\section{References}

1 Hong HS, Lee J, Lee E, Kwon YS, Lee E, Ahn W, Jiang MH, Kim JC and Son Y: A new role of substance P as an injuryinducible messenger for mobilization of CD29(+) stromal-like cells. Nat Med 15(4): 425-435, 2009. PMID: 19270709. DOI: 10.1038/nm.1909

2 Momin EN, Vela G, Zaidi HA and Quiñones-Hinojosa A: The oncogenic potential of mesenchymal stem cells in the treatment of cancer: Directions for future research. Curr Immunol Rev 6(2): 137-148, 2010. PMID: 20490366. DOI: $10.2174 / 157339510791111718$ 
3 Dvorak HF: Tumors: wounds that do not heal. Similarities between tumor stroma generation and wound healing. N Engl J Med 315(26): 1650-1659, 1986. PMID: 3537791. DOI: 10.1056/ NEJM198612253152606

4 Rhee KJ, Lee JI and Eom YW: Mesenchymal stem cell-mediated effects of tumor support or suppression. Int J Mol Sci 16(12): 30015-30033, 2015. PMID: 26694366. DOI: 10.3390/ijms 161226215

5 Liang W, Chen X, Zhang S, Fang J, Chen M, Xu Y and Chen X: Mesenchymal stem cells as a double-edged sword in tumor growth: focusing on MSC-derived cytokines. Cell Mol Biol Lett 26(1): 3 , 2021. PMID: 33472580. DOI: 10.1186/s11658-020-00246-5

6 Delinasios JG, Angeli F, Koumakis G, Kumar S, Kang WH, Sica G, Iacopino F, Lama G, Lamprecht S, Sigal-Batikoff I, Tsangaris GT, Farfarelos CD, Farfarelos MC, Vairaktaris E, Vassiliou S and Delinasios GJ: Proliferating fibroblasts and HeLa cells cocultured in vitro reciprocally influence growth patterns, protein expression, chromatin features and cell survival. Anticancer Res 35(4): 1881-1916, 2015. PMID: 25862842.

7 Jafari A, Rezaei-Tavirani M, Farhadihosseinabadi B, Zali H and Niknejad H: Human amniotic mesenchymal stem cells to promote/suppress cancer: two sides of the same coin. Stem Cell Res Ther 12(1): 126, 2021. PMID: 33579346. DOI: 10.1186/s13287-021-02196-x

8 Jung PY, Ryu H, Rhee KJ, Hwang S, Lee CG, Gwon SY, Kim J, Kim J, Yoo BS, Baik SK, Bae KS and Eom YW: Adipose tissue-derived mesenchymal stem cells cultured at high density express IFN- $\beta$ and TRAIL and suppress the growth of H460 human lung cancer cells. Cancer Lett 440-441: 202-210, 2019. PMID: 30393160. DOI: 10.1016/j.canlet.2018.10.017

9 Fakiruddin KS, Ghazalli N, Lim MN, Zakaria Z and Abdullah $\mathrm{S}$ : Mesenchymal stem cell expressing TRAIL as targeted therapy against sensitised tumour. Int J Mol Sci 19(8): 2188, 2018. PMID: 30060445. DOI: 10.3390/ijms 19082188

10 Salmasi Z, Hashemi M, Mahdipour E, Nourani H, Abnous K and Ramezani M: Mesenchymal stem cells engineered by modified polyethylenimine polymer for targeted cancer gene therapy, in vitro and in vivo. Biotechnol Prog 36(6): e3025, 2020. PMID: 32410328. DOI: $10.1002 /$ btpr.3025

11 Hamann A, Nguyen A and Pannier AK: Nucleic acid delivery to mesenchymal stem cells: a review of nonviral methods and applications. J Biol Eng 13: 7, 2019. PMID: 30675180. DOI: 10.1186/s13036-019-0140-0

12 Loebinger MR, Sage EK, Davies D and Janes SM: TRAILexpressing mesenchymal stem cells kill the putative cancer stem cell population. Br J Cancer 103(11): 1692-1697, 2010. PMID: 21063402. DOI: $10.1038 /$ sj.bjc.6605952

13 Sasportas LS, Kasmieh R, Wakimoto H, Hingtgen S, van de Water JA, Mohapatra G, Figueiredo JL, Martuza RL, Weissleder $\mathrm{R}$ and Shah K: Assessment of therapeutic efficacy and fate of engineered human mesenchymal stem cells for cancer therapy. Proc Natl Acad Sci USA 106(12): 4822-4827, 2009. PMID: 19264968. DOI: 10.1073/pnas.0806647106

14 van Boxel-Dezaire AH, Rani MR and Stark GR: Complex modulation of cell type-specific signaling in response to type I interferons. Immunity 25(3): 361-372, 2006. PMID: 16979568. DOI: $10.1016 /$ j.immuni.2006.08.014

15 Chawla-Sarkar M, Leaman DW and Borden EC: Preferential induction of apoptosis by interferon (IFN)-beta compared with IFN-alpha2: correlation with TRAIL/Apo2L induction in melanoma cell lines. Clin Cancer Res 7(6): 1821-1831, 2001. PMID: 11410525.

16 Stark GR, Kerr IM, Williams BR, Silverman RH and Schreiber RD: How cells respond to interferons. Annu Rev Biochem 67: 227-264, 1998. PMID: 9759489. DOI: 10.1146/annurev. biochem.67.1.227

17 Chawla-Sarkar M, Leaman DW, Jacobs BS and Borden EC: IFN-beta pretreatment sensitizes human melanoma cells to TRAIL/Apo2 ligand-induced apoptosis. J Immunol 169(2): 847855, 2002. PMID: 12097388. DOI: 10.4049/jimmunol.169.2.847

18 Chawla-Sarkar M, Lindner DJ, Liu YF, Williams BR, Sen GC, Silverman RH and Borden EC: Apoptosis and interferons: role of interferon-stimulated genes as mediators of apoptosis. Apoptosis 8(3): 237-249, 2003. PMID: 12766484. DOI: 10.1023/a:1023668705040

19 Zuk PA, Zhu M, Mizuno H, Huang J, Futrell JW, Katz AJ, Benhaim P, Lorenz HP and Hedrick MH: Multilineage cells from human adipose tissue: implications for cell-based therapies. Tissue Eng 7(2): 211-228, 2001. PMID: 11304456. DOI: $10.1089 / 107632701300062859$

20 Oggu GS, Sasikumar S, Reddy N, Ella KKR, Rao CM and Bokara KK: Gene delivery approaches for mesenchymal stem cell therapy: Strategies to increase efficiency and specificity. Stem Cell Rev Rep 13(6): 725-740, 2017. PMID: 28815481. DOI: $10.1007 / \mathrm{s} 12015-017-9760-2$

21 Baldo A, van den Akker E, Bergmans HE, Lim F and Pauwels $\mathrm{K}$ : General considerations on the biosafety of virus-derived vectors used in gene therapy and vaccination. Curr Gene Ther 13(6): 385-394, 2013. PMID: 24195604. DOI: 10.2174/1566523 2113136660005

22 Barkholt L, Flory E, Jekerle V, Lucas-Samuel S, Ahnert P, Bisset L, Büscher D, Fibbe W, Foussat A, Kwa M, Lantz O, Mačiulaitis R, Palomäki T, Schneider CK, Sensebé L, Tachdjian G, Tarte K, Tosca L and Salmikangas P: Risk of tumorigenicity in mesenchymal stromal cell-based therapies-bridging scientific observations and regulatory viewpoints. Cytotherapy 15(7): 753759, 2013. PMID: 23602595. DOI: 10.1016/j.jcyt.2013.03.005

23 Wuchter P, Bieback K, Schrezenmeier H, Bornhäuser M, Müller LP, Bönig H, Wagner W, Meisel R, Pavel P, Tonn T, Lang P, Müller I, Renner M, Malcherek G, Saffrich R, Buss EC, Horn P, Rojewski M, Schmitt A, Ho AD, Sanzenbacher R and Schmitt M: Standardization of Good Manufacturing Practice-compliant production of bone marrow-derived human mesenchymal stromal cells for immunotherapeutic applications. Cytotherapy 17(2): 128-139, 2015. PMID: 24856898. DOI: 10.1016/j.jcyt. 2014.04.002

24 Foster GR, Rodrigues O, Ghouze F, Schulte-Frohlinde E, Testa D, Liao MJ, Stark GR, Leadbeater L and Thomas HC: Different relative activities of human cell-derived interferon-alpha subtypes: IFN-alpha 8 has very high antiviral potency. J Interferon Cytokine Res 16(12): 1027-1033, 1996. PMID: 8974005. DOI: 10.1089/jir.1996.16.1027

25 Rani MR and Ransohoff RM: Alternative and accessory pathways in the regulation of IFN-beta-mediated gene expression. J Interferon Cytokine Res 25(12): 788-798, 2005. PMID: 16375607. DOI: 10.1089/jir.2005.25.788

26 Ryu H, Oh JE, Rhee KJ, Baik SK, Kim J, Kang SJ, Sohn JH, Choi E, Shin HC, Kim YM, Kim HS, Bae KS and Eom YW: Adipose tissue-derived mesenchymal stem cells cultured at high density express IFN- $\beta$ and suppress the growth of MCF-7 human 
breast cancer cells. Cancer Lett 352(2): 220-227, 2014. PMID: 25016057. DOI: $10.1016 /$ j.canlet.2014.06.018

27 Hacein-Bey-Abina S, Hauer J, Lim A, Picard C, Wang GP, Berry CC, Martinache C, Rieux-Laucat F, Latour S, Belohradsky BH, Leiva L, Sorensen R, Debré M, Casanova JL, Blanche S, Durandy A, Bushman FD, Fischer A and Cavazzana-Calvo M: Efficacy of gene therapy for X-linked severe combined immunodeficiency. N Engl J Med 363(4): 355-364, 2010. PMID: 20660403. DOI: 10.1056/NEJMoa1000164

28 Wang W, Xu X, Li Z, Lendlein A and Ma N: Genetic engineering of mesenchymal stem cells by non-viral gene delivery. Clin Hemorheol Microcirc 58(1): 19-48, 2014. PMID: 25227201. DOI: $10.3233 / \mathrm{CH}-141883$
29 Foldvari M, Chen DW, Nafissi N, Calderon D, Narsineni L and Rafiee A: Non-viral gene therapy: Gains and challenges of noninvasive administration methods. J Control Release 240: 165190, 2016. PMID: 26686079. DOI: 10.1016/j.jconrel.2015. 12.012

Received April 30, 2021

Revised June 2, 2021

Accepted June 4, 2021 